\title{
Relationship of Frontal Cortex Glucose Metabolism with Depression-Anxiety Status in Patients with Early Stage Non-Small-Cell Lung Cancer: A Cross-Sectional Study
}

\author{
๑ Ertan Sahin, ๑ Gulcin Elboga*, ๑ Umut Elboga, ๑ Yusuf Zeki Celen \\ Gaziantep University Hospital, Clinic of Nuclear Medicine, Gaziantep, Turkey \\ *Gaziantep University Hospital, Clinic of Psychiatry, Gaziantep, Turkey
}

Abstract

Aim: We investigated the relationship between frontal cortex 18-fluorodeoxyglucose $\left({ }^{18} \mathrm{FDG}\right)$ uptake levels and depression-anxiety status in patients with early-stage non-small-cell lung cancer (E-NSCLC) who underwent ${ }^{18} \mathrm{FDG}$-positron emission tomography/computed tomography $\left({ }^{18} \mathrm{FDG}-\mathrm{PET} / \mathrm{CT}\right)$ imaging.

Methods: This prospective study was conducted between the May-November 2020, after the permission of ethics committee. A total of 34 patients with E-NSCLC who underwent ${ }^{18} \mathrm{FDG}-\mathrm{PET} / \mathrm{CT}$ imaging for diagnosis-staging and the control group consisting of 25 subjects were included in the study. Regional cerebral ${ }^{18}$ FDG uptake level was calculated by SUVmean. Depression-anxiety levels were determined using Hospital Anxiety and Depression Scale (HADS). The frontal cortex SUVmean ratio and depression-anxiety levels was compared between the groups, and the relationship between SUVmean ratio and depression-anxiety levels were performed.

Results: The frontal cortex SUVmean ratio values were somewhat lower in the patient group compared to the control group for both sexes, being more prominent in males. However, these differences were not statistically significant for both sexes [for male: $0.75 \pm 0.006,0.79 \pm 0.03 ;(p=0.055)$; for female: $0.73 \pm 0.04,0.74 \pm 0.049 ;(p=0.71)]$. In addition, a statistically significant weak negative correlation was found between frontal SUVmean values and anxiety levels in male patients $(r=-0.4697, p=0.01)$.

Conclusions: We believe that the measurement of frontal cortex ${ }^{18} \mathrm{FDG}$ uptake level and notification of the clinician about the patients with decreased values are important in patients with E-NSCLC who underwent ${ }^{18} \mathrm{FDG}$-PET/CT imaging.

Keywords: Positron emission tomography/computed tomography, carcinoma, non-small-cell lung, depression, anxiety

\section{Introduction}

Depression is known to be more commonly seen in those with cancer or other chronic additional diseases $(1,2)$. More common depressive disorders have been reported to occur especially in pancreas, breast and lung cancers (2). Although there is many evaluation scales that can be used for the diagnosis and screening of depression such as beck depression test, Hospital Anxiety and Depression Scale (HADS), patient health questionnaire (DSM-MD4), center for epidemiologic studies depression scale (CES-D), Hamilton Depression Rating Scale; since depression symptoms are similar to the general condition in cancer patients, it may be difficult to diagnose. The treatment of accompanying depression has been reported to provide a positive contribution on the prognosis and survival in cancer patients (2-4).

18-fluorodeoxyglucose-positron emission tomography/ computed tomography ( $\left.{ }^{18} \mathrm{FDG}-\mathrm{PET} / \mathrm{CT}\right)$ is an imaging method evaluating glucose metabolism, and today is widely used in many clinical indications, especially in the field of oncology. Since the main energy need of the cerebral tissue is glucose, and because physiologically high level of ${ }^{18} \mathrm{FDG}$ uptake is seen, most centers perform the imaging from the skull base in the oncologic ${ }^{18} \mathrm{FDG}-\mathrm{PET} /$

Address for Correspondence: Ertan Sahin, Gaziantep University Hospital, Clinic of Nuclear Medicine, Gaziantep, Turkey 
CT studies. But some centers as in our center perform the imaging from the vertex and the cerebral tissue is included in the imaging area. In this way, some incidental cerebral-cerebellar lesions and diseases may be detected in oncologic ${ }^{18} \mathrm{FDG}-\mathrm{PET} / \mathrm{CT}$ examines.

Although there are studies showing high frequency of depression in cancer and negative effects on prognosissurvival, there are few studies examining brain ${ }^{18} \mathrm{FDG}$ uptake levels in lung cancer $(5,6)$. In addition, looking at the literature, no study was found investigating the relationship between the presence of depression and cerebral ${ }^{18} \mathrm{FDG}$ uptake levels in early-stage (Stages 1-2) lung cancer (E-NSCLC).

In the light of this information, we aimed to investigate the relationship between frontal cortex ${ }^{18} \mathrm{FDG}$ uptake level and depression-anxiety status in patients who underwent ${ }^{18} \mathrm{FDG}-\mathrm{PET} / \mathrm{CT}$ imaging and were clinically accepted to have E-NSCLC, to compare the results with the control group and to evaluate whether ${ }^{18}$ FDG uptake can be a helpful parameter in predicting the diagnosis of depression in E-NSCLC.

\section{Methods}

\section{Subjects}

The patient information form was filled in for this study and the approval of the institutional ethics committee was obtained from Gaziantep University (number; 2020-152, date; 15/04/020). This prospective study was conducted between May and November 2020, after the permission of the ethics committee. A total of 34 patients with 25 being males and 9 females who underwent ${ }^{18} \mathrm{FDG}-\mathrm{PET} / \mathrm{CT}$ for the diagnosis and/or staging in our clinic and accepted to have early-stage (Stages 1-2) lung cancer ${ }^{18}$ FDG-PET/ CT and chest CT findings were included in the study. In addition, a total of 25 persons with 16 being males and 9 females who underwent ${ }^{18} \mathrm{FDG}-\mathrm{PET} / \mathrm{CT}$ due to any cancer suspicion in our clinic and without any malignancy detected were assigned to the control group. When establishing the control group, it was paid attention to be similar to the patient groups' features as age, gender, profession, educational status.

Newly diagnosed patients who did not receive any treatment (radiotherapy, chemotherapy, surgery), were histopathologically diagnosed with non-small-cell lung cancer (NSCLC), and accepted as to have early-stage (Stages 1-2) lung carcinoma on ${ }^{18} \mathrm{FDG}$-PET/CT and chest CT findings, and persons without any pathological ${ }^{18} \mathrm{FDG}$ uptake detected as the control group were included in the study.

Patients with a known pathology/drug use/surgery that could affect cerebral-cerebellar ${ }^{18}$ FDG uptake, pregnant and breastfeeding patients and those aged under 18 years were excluded from the study.

\section{Patient Preparation and ${ }^{18} \mathrm{FDG}-\mathrm{PET} / \mathrm{CT}$ Imaging Protocol}

The blood glucose of the patients was cotrolled the following 12-hour fasting and if glucose level $<150 \mathrm{mg} /$ $\mathrm{dL}$, were injected with $5 \mathrm{MBq} / \mathrm{Kg}$ intravenous (iv) ${ }^{18} \mathrm{FDG}$.

The PET/CT imaging was performed 60 minutes after the injection using combined PET/CT device (Biograph2 LSO/Somatom Emotion; Siemens Medical Solutions) in our department.

The imaging was performed as whole-body from vertex up to the upper thigh region at mean 5-7 bed interval.

\section{Imaging Analysis and Evaluation of the Patients}

${ }^{18}$ FDG-PET/CT images of the patient and control groups included in the study were evaluated by two experienced nuclear medicine specialists. Frontal cortex ${ }^{18} \mathrm{FDG}$ uptake level was found by calculated by averaging ratios of the mean standard uptake values (SUVmean) taken from the superior, middle and inferior levels of the frontal cortex area to the SUVmean measurements taken from the other cerebral regions in similar sections.

Liver SUVmax values were also measured in order to test the possible variabilities related to the other factors that could affect FDG uptake in the body.

Depression-anxiety levels of the persons in the patient and control groups who met the inclusion criteria were determined 2 days after ${ }^{18}$ FDG-PET/CT imaging using HADS, which validity and reliability was studies by Aydemir et al. (7), by a psychiatrist who was blind to ${ }^{18} \mathrm{FDG}$-PET/CT findings.

The HADS scale consisted of 14 questions with anxiety was measured with seven (uneven-number items: 1, 3, 5, 7, 9, 11 and 13) and depression with seven (even-number items: 2, 4, 6, 8, 10, 12 and 14) questions. The answers were scored with 0-3 points and the minimum score that can be obtained from the scale was 0 and the maximum score was 21 points. As specified in the study by Aydemir et al. (7), cut-off points for the Turkish form of the scale was taken as 10 points for anxiety (HADS-A) and 7 for depression (HADS-D).

Both groups were examined in terms of age, gender, profession, educational level, blood glucose, frontal cortex SUVmean ratio, depression and anxiety levels.

The relationship between frontal cortex SUV mean ratio and depression-anxiety levels was analyzed.

\section{Statistical Analysis}

Data of the patient and control groups were obtained via the medical history, HADS scale and ${ }^{18}$ FDG-PET/CT images. Data were analyzed using SPSS v.18 statistical software. Mean, standard deviation and percentage descriptive values were used for age, blood glucose levels, liver SUVmax, frontal cortex SUVmean and HADS scale 
results. Quantitative variables were expressed as mean \pm standard deviation (SD) when normally distributed, and as [IQR, (interquartile range)] when non-normally distributed. The comparison between the groups was made with t-tests, Fisher Exact and chi-square tests. Pearson's analysis was used to determine the correlation between frontal cortex SUVmean values and HADS scores. The $p<0.05$ were considered statically significant for all statistical tests.

\section{Results}

Demographic features (age, gender, profession, educational status), frontal cortex SUVmean, HADS-A and HADS-D results of the patient and control groups, intergroup comparisons of these parameters and statistical analysis data are given in Table 1,2, and these data were explained shortly with the following sentences.

There was no statistical difference between the two groups in terms of age, gender, profession, educational status, blood glucose levels and liver SUVmax values $(p>0.05)$.

Frontal cortex SUVmean ratios were slightly lower in both sexes in the patient group compared to the control group, although the difference was not statistically significant $(p>0.05)$.

The correlation between frontal cortex SUVmean values and HADS-A and HADS-D scores was analyzed in the patient group, and a statistically significant weak negative correlation was found between frontal cortex SUVmean values and HADS-A in the male patients group ( $r=-0.4697$, $p=0.01$ ), while no statistically significant correlation was found in the other sex and scores.
Figure 1 shows fusion ${ }^{18} \mathrm{FDG}-\mathrm{PET} / \mathrm{CT}$ images of a patient with decreased frontal cortex FDG uptake (SUVmean ratio: 0.68) compared to the control group who a HADS-A score of 12 and a HADS-D score of 9, while Figure 2 shows fusion ${ }^{18} \mathrm{FDG}-\mathrm{PET} / \mathrm{CT}$ images of a patient with normal frontal cortex FDG uptake (SUVmean ratio:
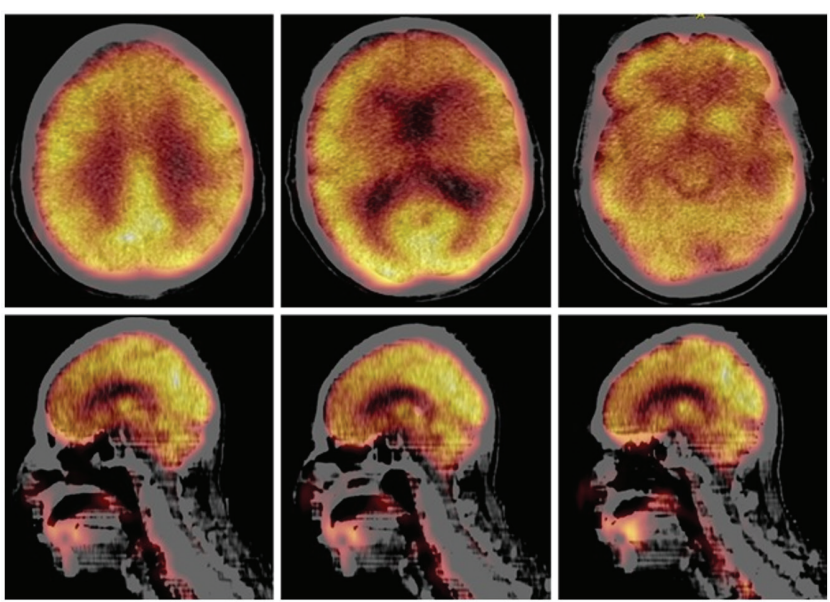

Figure 1. Cranial FDG-PET/CT fusion images of a 59-yearold male patient (top row: transaxial sections from the vertex towards inferior; bottom row: sagittal sections): Frontal cortex FDG uptake appears to be decreased (Frontal cortex SUVmean $r=0.68)$. HADS-A and HADS-D scores were found 12 and 9, respectively.

FDG: Fluorodeoxyglucose, PET: Positron emission tomography, CT: Computed tomography, SUV: Standart uptake value, HADS-A: Hospital anxiety and depression scale-anxiety, HADS-D: Hospital anxiety and depression scale-depression

\begin{tabular}{|l|l|l|l|l|l|l|}
\hline Table 1. The Patient and control groups' characteristics \\
\hline Characteristics & $\begin{array}{l}\text { Male } \\
\text { patients }\end{array}$ & $\begin{array}{l}\text { Male } \\
\text { control }\end{array}$ & $\mathbf{p}$ & $\begin{array}{l}\text { Female } \\
\text { patients }\end{array}$ & $\begin{array}{l}\text { Female } \\
\text { control }\end{array}$ & p \\
\hline Subject number & 25 & 16 & $p^{*}=0.56$ & 9 & 9 & $p^{*}=0.56$ \\
\hline Age (year) & $57.8 \pm 7.2$ & $55.1 \pm 5.9$ & $p^{* *}=0.22$ & $53.8 \pm 7.8$ & $52.7 \pm 6.6$ & $p^{* *}=0.74$ \\
\hline Education level $(\downarrow / \uparrow)$ & $12 / 13$ & $6 / 10$ & $p^{*}=0.53$ & $7 / 2$ & $6 / 3$ & $p^{* * *}=0.53$ \\
\hline Profession (working or retired/not working) & $24 / 1$ & $15 / 1$ & $p^{*}=1.0$ & $8 / 1$ & $7 / 2$ & $p^{* * *}=0.52$ \\
\hline Education level $\downarrow$ : High school or below, Education level $\uparrow:$ University, $p^{*}$ : Fisher's Exact test, $p^{* *}$ : T-test, $p^{* * *}$ : Chi-square test &
\end{tabular}

Table 2. The Inter-group comparison for the metabolic findings and anxiety-depression scores

\begin{tabular}{|l|l|l|l|l|l|l|}
\hline Data & Male patients & $\begin{array}{l}\text { Male } \\
\text { control }\end{array}$ & $\mathbf{p}$ & $\begin{array}{l}\text { Female } \\
\text { patients }\end{array}$ & $\begin{array}{l}\text { Female } \\
\text { control }\end{array}$ & $\mathbf{p}$ \\
\hline Glucose level $(\mathrm{mg} / \mathrm{dL})$ & $117.8 \pm 12.5$ & $112.9 \pm 14.0$ & $\mathrm{p}^{*}=0.25$ & $113.3 \pm 12.4$ & $111.7 \pm 13.7$ & $\mathrm{p}^{*}=0.80$ \\
\hline Liver SUVmax & $3.37 \pm 0.43$ & $3,28 \pm 0.51$ & $\mathrm{p}^{*}=0.27$ & $3.30 \pm 0.37$ & $3.23 \pm 0.36$ & $\mathrm{p}^{*}=0.35$ \\
\hline Frontal cortex SUVmean ratio & $0.75 \pm 0.06$ & $0.79 \pm 0.03$ & $\mathrm{p}^{*}=0.055$ & $0.73 \pm 0.04$ & $0.74 \pm 0.04$ & $\mathrm{p}^{*}=0.71$ \\
\hline HADS-A score & $9.7 \pm 3.6$ & $8.5 \pm 2.7$ & $\mathrm{p}^{*}=0.28$ & $10.8 \pm 4.3$ & $9.2 \pm 2.5$ & $\mathrm{p}^{*}=0.33$ \\
\hline HADS-D score & $5.8 \pm 1.7$ & $5.1 \pm 1.5$ & $\mathrm{p}^{*}=0.18$ & $7.3 \pm 2.0$ & $6.8 \pm 1.7$ & $\mathrm{p}^{*}=0.62$ \\
\hline Correlation of SUVmean and HADS-A & $\mathrm{r}=-0.4697$ & - & $\mathrm{p}^{* *}=0.01$ & $\mathrm{r}=-0.4813$ & - & $\mathrm{p}^{* *}=0.18$ \\
\hline Correlation of SUVmean and HADS-D & $\mathrm{r}=-0.3754$ & - & $\mathrm{p}^{* *}=0.06$ & $\mathrm{r}=-0.5303$ & - & $\mathrm{p}^{* *}=0.14$ \\
\hline
\end{tabular}

SUV: Standart uptake value, HADS-A: Hospital anxiety and depression scale-anxiety, HADS-D: Hospital anxiety and depression scale-depression, $\mathrm{p}^{\star}$ : T-test, $\mathrm{p}^{\star \star}$ : Pearson correlation analysis 
0.81) compared to the control group who a HADS-A score of 9 and a HADS-D score of 6 .
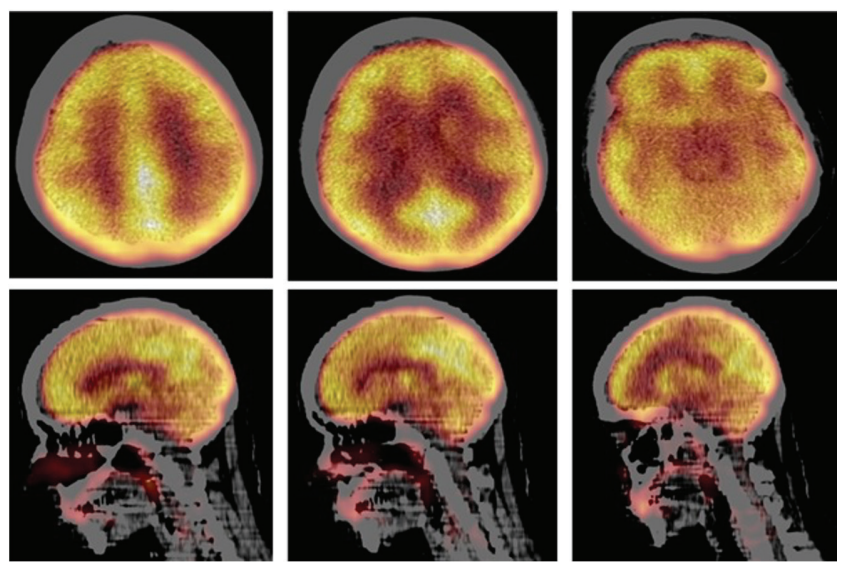

Figure 2. Cranial FDG-PET/CT fusion images of a 46-year-old female patient (top row: transaxial sections from the vertex towards inferior; bottom row: sagittal sections): There is no decrease in frontal cortex FDG uptake and it is appear to be normally (Frontal cortex SUVmean $r=0.81$ ). HADS-A and HADS-D scores were found 9 and 6, respectively.

FDG: Fluorodeoxyglucose, PET: Positron emission tomography, CT: Computed tomography, SUV: Standart uptake value, HADS-A: Hospital anxiety and depression scale-anxiety, HADS-D: Hospital anxiety and depression scale-depression

\section{Discussion}

Although establishing the diagnosis of depression is difficult in cancer patients due to similarity between general status features and depression symptoms, there are studies in the literature demonstrating high incidence and negative effects of depression on prognosis in patients with lung cancer (3,8-11). On the other hand, the number of studies investigating cerebral FDG uptake levels and the relationship between the presence of depression and cerebral FDG uptake in patients with lung cancer is limited $(5,6)$. In our study, we compared frontal cortex FDG uptake between patients with non-small cell lung carcinoma and the control group, and investigated its relationship with HADS-A and HAS-D scores. In this respect, our study is the first in the literature.

Many factors such as age, gender, occupation, educational status, PET imaging procedure-parameters, blood glucose level, possible drugs used, neurologicalvascular-systemic diseases can cause changes in brain FDG uptake. In order to minimize these, maximum attention was paid to ensure inclusion-exclusion criteria of the study and to be no statistical differences between the patientcontrol group in terms of these factors.

Because the probability of unpredictable metastases to distant organs such as the brain in small cell lung carcinoma, and high probability of cerebral metastasis in advanced stage lung cancer, we were included only patients with E-NSCLC in our study. When reviewing of few studies $(5,6)$ which evaluating brain ${ }^{18} \mathrm{FDG}$ uptake in patients with lung cancer in the literature, it is seen that they are designed without regard to case discrimination, and therefore we believe that our study is superior in this sense.

Although ${ }^{18} \mathrm{FDG}$ uptake is physiologically high in the cerebral tissue, the imaging is started from the cranial vertex in ${ }^{18} \mathrm{FDG}$-PET/CT examinations in some centers, and although procedures-parameters in neuropsychiatric cerebral ${ }^{18} \mathrm{FDG}-\mathrm{PET} / \mathrm{CT}$ examinations are not used, incidental cerebral-cerebellar metastases, CVD and demantial pathologies may be detected. Similarly, we are routinely include the brain to imaging area on PET examinations and we would like to emphasize that no additional imaging was performed except for routine imaging in our study.

It is argued that regional increases and decreases in cerebral FDG intake of cancer patients are caused by the interaction between central neuromodulation and tumorassociated peripheral reaction. In the study conducted by Zhang et al. (5), brain regions where decreases/increases in FDG uptake in small cell lung cancer patients were identified and possible mechanisms were investigated. Whereas in our study only frontal lobe FDG uptake levels were investigated, and the other cerebral regions could not be examined. The most important reason for this was the lack of a software specific to cerebral studies such as cortexID and NeuroQuant, and not performed the neurologic PET/CT examination which containing differing imaging procedures-parameters.

Although evaluating only frontal lobe FDG uptake may be seen as a weak aspect of our study, there are studies showing that prefrontal dysfunction is the pathologic basis of depression onset and also a marker of a depressive condition $(12,13)$. In addition, it has been reported that frontal and parietal regions are the main components of the dorsal attention network (DAN), which controls attention allocation and spatial orientation functions, and these may be associated with possible dysfunction in cancer patients $(6,14,15)$. In the present study, although no statistically significant difference was not found between both sexes in the patient group compared to the control group in terms of frontal cortex FDG uptake, it was lower in the patient group than in the controls, and these findings were supporting the literature.

Clinical examination is necessary for the diagnosis of depression, although HADS is a simple, but simple, reliable 
depression evaluating tool designed for use in medical practice. Studies have shown that this scale can be used not only for hospital setting, but also social and first line medical applications. The reason for using this scale in our study was that the study group was in the hospital, the scale can be used in a practical way in about five minutes and validity-reliability study of the scale was conducted for our population.

HADS-A and HADS-D scores were found to be higher for both sexes in the patient group compared to the control group. However, these differences were not statistically significant. It should be taken into account that, this result might be resulted from that although the control group consisted of the persons without pathologic ${ }^{18} \mathrm{FDG}$ uptake detected, this group could not be created with completely healthy persons without health problems and/or our patient group consisted of the patients with early-stage (Stages 1-2) disease.

When the relationship between the frontal cortex SUVmean values, HADS-A and HADS-D scores was analyzed in the patient group, there was a statistically significant weak negative correlation between frontal cortex SUVmean values and HADS-A scores only in the male patients group. Although a weak correlation was found only in male patients group and the study group and assessment scale were different, in a study by Fu C et al. (16) it was reported that SUV values were low in ${ }^{18} \mathrm{FDG} /$ PET-CT imaging in the frontal gyrus region and showed correlation with the Hamilton score in major depressive patient compared to the control group.

Although the HADS scale has been performed 2 days after ${ }^{18} \mathrm{FDG} / \mathrm{PET}-\mathrm{CT}$ imaging in the patient and control groups opened the door for critical opinion that anxietydepression evaluation was not made concurrently, we think that this provided an advantage of ruling out concern-fear state that can be caused by the imaging process.

\section{Study Limitations}

Besides being the first prospective cross-sectional study conducted in this area, our study also has some main limitations. These can be listed as follows; the presence of depression could not be determined with clinical evaluation, some software evaluating cerebral metabolism in a more standardized way could not be used and the number of patients was relatively small.

\section{Conclusion}

We found that the frontal cortex ${ }^{18} \mathrm{FDG}$ uptake was lower in the patient group than in the controls and, there is a relationship between the frontal cortex SUVmean values and HADS-A scores in male patients. We think that it is important to measure frontal cortex ${ }^{18} \mathrm{FDG}$ uptake in NSLC patients who underwent ${ }^{18} \mathrm{FDG}-\mathrm{PET} / \mathrm{CT}$ imaging and to inform the clinician about the patients with decreased ${ }^{18} \mathrm{FDG}$ uptake values of the frontal cortex for the prediction of likely depressive status.

\section{Authorship Contributions}

Concept: E.S, Design: E.S., Data Collection or Processing: E.S., G.E, Analysis or Interpretation: E.S., G.E., U.E., Y.Z.C., Literature Search: E.S., Writing: E.S.

Conflict of Interest: No conflict of interest was declared by the authors.

Financial Disclosure: The authors declared that this study received no financial support.

\section{References}

1. Lyra V, Parissis J, Kallergi M, et al. ${ }^{18}$ F-FDG PET/CT brain glucose metabolism as a marker of different types of depression comorbidity in chronic heart failure patients with impaired systolic function. Eur J Heart Fail 2020;22:2138-46.

2. Sherrill C, Smith M, Mascoe C, Bigus E, Abbitt D. Effect of Treating Depressive Disorders on Mortality of Cancer Patients. Cureus 2017;9:1740.

3. Sullivan DR, Ganzini L, Duckart JP, et al. Treatment receipt and outcomes among lung cancer patients with depression. Clin Oncol (R Coll Radiol) 2014;26:25-31.

4. Walker AJ, Grainge M, Bates TE, Card TR. Survival of glioma and colorectal cancer patients using tricyclic antidepressants post-diagnosis. Cancer Causes Control 2012;23:1959-64.

5. Zhang W, Ning N, Li X, et al. Changes of Brain Glucose Metabolism in the Pretreatment Patients with Non-Small Cell Lung Cancer: A Retrospective PET/CT Study. PLoS One 2016;11:0161325.

6. Li WL, Fu C, Xuan A, et al. Preliminary study of brain glucose metabolism changes in patients with lung cancer of different histological types. Chin Med J (Engl) 2015;128:301-4.

7. Aydemir Ö, Güvenir T, Küey L, Kültür S. Hastane Anksiyete ve Depresyon Ölçeği Türkçe Formunun Geçerlik Güvenilirlik Çalışması. Turk Psikiyatri Derg 1997;8:197-280.

8. Wang YH, Li JQ, Shi JF, et al. Depression and anxiety in relation to cancer incidence and mortality: a systematic review and meta-analysis of cohort studies. Mol Psychiatry 2020;25:1487-99.

9. Pirl WF, Greer JA, Traeger L, et al. Depression and survival in metastatic non-small-cell lung cancer: effects of early palliative care. J Clin Oncol 2012;30:1310-5.

10. Sullivan DR, Forsberg CW, Ganzini L, et al. Longitudinal Changes in Depression Symptoms and Survival Among Patients With Lung Cancer: A National Cohort Assessment. J Clin Oncol 2016;34:3984-91.

11. McFarland DC, Saracino RM, Miller AH, Breitbart W, Rosenfeld B, Nelson C. Prognostic implications of depression and inflammation in patients with metastatic lung cancer. Future Oncol 2021;17:183-96. 
12. Kameyama M, Fukuda M, Yamagishi $Y$, et al. Frontal lobe function in bipolar disorder: a multichannel near-infrared spectroscopy study. Neuroimage 2006;29:172-84.

13. Lee HS, Choo IH, Lee DY, et al. Frontal Dysfunction Underlies Depression in Mild Cognitive Impairment: A FDG-PET Study. Psychiatry Investig 2010;7:208-14.

14. Ptak R, Schnider A. The dorsal attention network mediates orienting toward behaviorally relevant stimuli in spatial neglect. J Neurosci 2010;30:12557-65.

15. Nonokuma M, Kuwabara $Y$, Takano K, Yoshimitsu K. Demonstration of decrease in regional cerebral glucose metabolism in patients with lung cancer without apparent brain metastasis using statistical image analysis. J Nucl Med 2014;55:1596.

16. Fu C, Shi D, Gao Y, Xu J. Functional assessment of prefrontal lobes in patients with major depression disorder using a dual-mode technique of 3D-arterial spin labeling and ${ }^{18} \mathrm{~F}$-fluorodeoxyglucose positron emission tomography/ computed tomography. Exp Ther Med 2017;14:1058-64. 